\title{
Reply to Russo et al
}

Daniel Munblit ${ }_{1,2,3}$, Nikita A Nekliudov ${ }_{1}$, Polina Bugaeva 1 , Oleg Blyuss, ${ }_{1,4}$, Peter Timashev5,6,7, John O Warner, 2 , Pasquale Comberiatis, Christian Apfelbacher, ${ }_{9}$, Mikhail E Politovio, Andrey Yavorovskiy $_{10}$, Sergey Avdeevi1, Valentina A Kapustina12, Victor Fomin ${ }_{13}$, Andrey A Svistunovi3, Denis Butnaru $1_{13}$, Petr Glybochko ${ }_{13}$

1. Department of Paediatrics and Paediatric Infectious Diseases, Institute of Child"s Health, Sechenov First Moscow State Medical University (Sechenov University), Moscow, Russia

2. Inflammation, Repair and Development Section, National Heart and Lung Institute, Faculty of Medicine, Imperial College London, London, United Kingdom

3. Research and Clinical Center for Neuropsychiatry, Moscow, Russia

4. School of Physics, Astronomy and Mathematics, University of Hertfordshire, College Lane, Hatfield, United Kingdom

5. Institute for Regenerative Medicine, Sechenov First Moscow State Medical University (Sechenov University), Moscow, Russia

6. Chemistry Department, Lomonosov Moscow State University, Russia

7. Department of Polymers and Composites, N.N. Semenov Institute of Chemical Physics, Russia

8. Department of Clinical and Experimental Medicine, Section of Pediatrics, University of Pisa, Pisa, Italy

9. Institute of Social Medicine and Health Systems Research, Faculty of Medicine, Otto von Guericke

University Magdeburg, Magdeburg, Germany

10. Department of Intensive Care, Sechenov First Moscow State Medical University (Sechenov University), Moscow, Russia

11. Clinic of Pulmonology, Sechenov First Moscow State Medical University (Sechenov University), Moscow, Russia

12. Department of Internal Medicine №1, Institute of Clinical Medicine, Sechenov First Moscow State

Medical University (Sechenov University), Moscow, Russia

13. Sechenov First Moscow State Medical University (Sechenov University), Moscow, Russia

\section{Correspondence to}

Daniel Munblit: daniel.munblito8@imperial.ac.uk

\section{Conflict of Interest Information}

Authors have no conflict of interest to declare. We thank RFBR, grant 20-04-60063 for supporting the work.

\section{Word count: 467}


To the Editor,

We thank Dr. Russo and colleagues for taking the time to critically appraise and respond to our recent publication assessing clinical and laboratory characteristics of patients admitted to the Sechenov University hospital network in Moscow, Russia for suspected COVID-19 infection. We read about the findings of the RESILIENCY study [1] with great interest, as they are much in agreement with the results from our cohort. This further confirms the importance of appropriate clinical management of all admitted patients with suspected SARS-CoV-2 infection irrespective of the reverse transcription polymerase chain reaction (RT-PCR) result.

In their study, Russo et al [1] demonstrated that over half of the admitted patients did not have positive RT-PCR at the time of admission. The authors presented the clinical management protocol for all patients admitted to the emergency room (ER) with respiratory failure and/or fever who require management for suspected COVID-19. The findings are in line with the outcomes of our study, as $50.3 \%$ of patients admitted to hospital in our cohort did not have a single positive RT-PCR swab result. Likewise, the diagnostic and treatment strategies were based primarily on clinical and laboratory findings for all admitted patients, irrespective of the RT-PCR results.

A high false-negative rate of the RT-PCR tests, varying between 20 and 66\%, depending on the day since symptom onset has been previously reported [2]. Although negative RT-PCR tests were found in almost half of patients in a few large cohort studies [3-5], including our own, patients with suspected COVID-19 infection were normally excluded from statistical analysis in the absence of the positive test result [3, 4, 6-9]. We would like to emphasise that most of the national and international data on COVID-19 cases and deaths are based exclusively on positive RT-PCR and may seriously underestimate the true prevalence and mortality of COVID-19. There is a pressing need to account for the number of patients with clinical features of COVID-19 and negative RTPCR.

Russo et al. suggest that some clinical and laboratory features may help physicians discriminate cases of SARS-CoV-2 from other causes regardless of the RT-PCR results. We support authors in their initiative to develop reliable parameter sets to allow for early differentiation of patients at higher risk of unfavourable outcomes. This is very much in line with the national and international efforts to harmonise and collate data on clinical characteristics of COVID-19 and develop clinical algorithms and scoring systems. A recent multicentre study from the International Severe Acute Respiratory and emerging Infections Consortium Coronavirus Clinical Characterisation Consortium-(ISARIC-4C) resulted in the development of a very promising pragmatic risk score to predict mortality in patients admitted to hospital with COVID-19 [10], demonstrating an excellent negative predictive value. Future research should focus on the development and validation of reliable and user-friendly tools for use in routine clinical practice. 


\section{References}

1. Russo A, Bellelli V, Ceccarelli G, et al. Comparison between hospitalized patients affected or not by COVID-19 (RESILIENCY study). Clin Infect Dis 2020.

2. Kucirka LM, Lauer SA, Laeyendecker O, Boon D, Lessler J. Variation in False-Negative Rate of Reverse Transcriptase Polymerase Chain Reaction-Based SARS-CoV-2 Tests by Time Since Exposure. Ann Intern Med 2020.

3. Argenziano MG, Bruce SL, Slater CL, et al. Characterization and clinical course of 1000 Patients with COVID-19 in New York: retrospective case series. medRxiv 2020.

4. Petrilli CM, Jones SA, Yang J, et al. Factors associated with hospital admission and critical illness among 5279 people with coronavirus disease 2019 in New York City: prospective cohort study. BMJ 2020; 369: m1966.

5. Munblit D, Nekliudov NA, Bugaeva P, et al. StopCOVID cohort: An observational study of 3,480 patients admitted to the Sechenov University hospital network in Moscow city for suspected COVID-19 infection. Clin Infect Dis 2020.

6. Docherty AB, Harrison EM, Green CA, et al. Features of 20133 UK patients in hospital with covid19 using the ISARIC WHO Clinical Characterisation Protocol: prospective observational cohort study. BMJ 2020; 369: m1985.

7. Grasselli G, Zangrillo A, Zanella A, et al. Baseline Characteristics and Outcomes of 1591 Patients Infected With SARS-CoV-2 Admitted to ICUs of the Lombardy Region, Italy. JAMA 2020.

8. Richardson S, Hirsch JS, Narasimhan M, et al. Presenting Characteristics, Comorbidities, and Outcomes Among 5700 Patients Hospitalized With COVID-19 in the New York City Area. JAMA 2020.

9. Zhou F, Yu T, Du R, et al. Clinical course and risk factors for mortality of adult inpatients with COVID-19 in Wuhan, China: a retrospective cohort study. Lancet 2020; 395(10229): 1054-62.

10. Knight SR, Ho A, Pius R, et al. Risk stratification of patients admitted to hospital with covid-19 using the ISARIC WHO Clinical Characterisation Protocol: development and validation of the $4 C$ Mortality Score. BMJ 2020; 370: m3339. 